\title{
LOS ORÍGENES FILOSÓFICOS DE LA NOCIÓN DE SOBERANÍA NACIONAL EN EL CONTRACTUALISMO político de Thomas HobBes, John LOCKE Y JEAN-JACQUES RousSEAu
}

[The Phylosophical Origins of the Notion of National Sovereignity in the Political Contractualism of Thomas Hobbes, John Locke and Jean-Jacques Rousseau]

\author{
Anderson Vichinkeski TeIXeIRA* \\ Universidade do Vale do Rio dos Sinos
}

\begin{abstract}
RESUMEN
El artículo examina cómo las proposiciones teóricas de Thomas Hobbes, John Locke y Jean-Jacques Rousseau sobre la función del contrato social han tenido una profunda conexión con la noción de soberanía nacional, desde su fundamentación filosófica hasta sus límites. Para eso, se estudia en los tres filósofos el estado de naturaleza y la concepción de hombre, el proceso de formación del contrato social y la condición del hombre en el Estado, según las formas de Estado y de Gobierno preferidas de los autores en estudio. A modo de conclusión, se busca reconstruir
\end{abstract}

Abstract

This article analyses, from the philosophical basis to its limits, the way in which the theoretical propositions of Thomas Hobbes, John Locke and Jean-Jacques Rousseau about the social contract function have been deeply connected to the notion of national sovereignty. To do so, and based on the forms of State and Government preferred by each of the three authors studied, the state of nature, the conception of man, the process of forming a social contract and the condition of mankind within the State are studied. The conclusion at-

ReCIBIDO el 1 de agosto y ACEPTADo el 22 de diciembre de 2014

* Doctor en teoría y historia del derecho por la Università degli Studi di Firenze, magíster en derecho del Estado (PUC/RS). Profesor del Programa de Postgrado en Derecho de la Universidade do Vale do Rio dos Sinos. Dirección postal: Av. Unisinos, 950, CCJ4, Sala 4A425, CEP 93.022-000, São Leopoldo/RS, Brasil. Dirección de correo electrónico: andersonvteixeira@hotmail.com 
la génesis de las más importantes características de la soberanía nacional.

\section{Palabras Clave}

Thomas Hobbes - John Locke - JeanJacques Rousseau - Contractualismo - Soberanía. tempts to reconstruct the genesis of the most important characteristics of national sovereignty.

\section{KEYWORDS}

Thomas Hobbes - John Locke Jean-Jacques Rousseau - Contractualism - Sovereignty.

\section{INTRODUCCIÓN}

En la filosofía política vemos, en un primer momento, los orígenes del concepto de soberanía estatal remontar al pensamiento político de Nicolás Maquiavelo, con su Il principe (1513). En un segundo momento, en el mismo siglo, Jean Bodin, con su Les siz livres de la Republique (1576), aparece como uno de los padres del concepto moderno de soberanía. Sin embargo, los dos autores no constituyen una tradición filosófica común y sus aportes teóricos buscaban, en realidad, afirmar el poder político contra el poder de la Iglesia. Véase que Maquiavelo pensaba el Estado como dominio, posesión y propiedad sobre un territorio ${ }^{1}$, mientras que Bodin introdujo la idea de unicidad de la soberanía, afirmando que la "souveraineté est la puissance absolue et perpétuelle d'une République"2.

Aunque Maquiavelo y Bodin sean los dos autores más célebres relacionados con los orígenes del concepto de soberanía, debemos centrarnos, en el presente artículo, en la tradición político-filosófica que más contribuciones dejó acerca de la génesis del concepto en cuestión: el contractualismo político.

Las proposiciones teóricas de Hobbes, Locke y Rousseau sobre la función del contrato social tienen una profunda conexión con la noción de soberanía nacional, desde su fundamentación filosófica hasta sus límites. Por lo tanto, las influencias del contractualismo estaban presentes ya en los siglos XVIII y XIX, sentando las bases filosóficas para el surgimiento de la idea de soberanía nacional. El acontecimiento político con más importantes repercusiones para la formación del concepto en cuestión fue, sin dudas, la Revolución Francesa. Sin embargo, el contractualismo político fue responsable por producir la base filosófica que permitió al concepto de soberanía estatal perder

${ }^{1}$ Ménessier, Thierry, Principauté et souveraineté chez Machiavel, en CazzaniGA, Gian Mario - Zarka, Yves-Charles (editores), Penser la souveraineté (Paris, Vrin, 2001), p. 29.

${ }^{2}$ Bodin, Jean, Les six livres de la République (Geneve, 1629), cap. $8^{\circ}$. 
sus orígenes absolutistas y tomar un enfoque basado en la idea de Estado moderno ${ }^{3}$. Como veremos, incluso un filósofo del absolutismo monárquico como Hobbes realizó aportes que culminaron en la justificación filosófica de la noción de soberanía nacional ${ }^{4}$.

Partiendo de una metodología histórico-analítico, buscaremos desarrollar el objetivo central de este trabajo en cuatro momentos: analizar cómo el estado de naturaleza y la concepción de hombre difieren en Hobbes, Locke y Rousseau (I); reconstruir el proceso de formación del contrato social en los autores examinados (II); verificar las formas de Estado y de Gobierno en los autores, sobre todo con relación a la condición del hombre en el Estado (III); y sintetizar, conclusivamente, la génesis de las características de la soberanía nacional ()IV).

\section{El ESTAdo DE NATURALEZA COMO CATEGORÍA TEÓRICO-HIPOTÉTICA}

Para analizar las concepciones de hombre en los autores en cuestión debemos considerar, inicialmente, el momento en el que los hombres no tenían la fuerza del poder político sobre sí mismos y ni siquiera la sociedad civil estaba formada, es decir, iniciamos en el estado de naturaleza. Se podría argumentar prima facie que para Hobbes y Locke esa cuestión no es muy controvertida, ya que ambos consideraban que ese había sido el momento anterior a la formación del contrato social, momento en el que los hombres se reunían en plena libertad de acción y según la más pura igualdad natural. Pero, si analizamos más detenidamente ambas teorías, vemos que las razones por las que los hombres deben contratar son completamente diferentes en esos dos autores.

En Hobbes, la inseguridad constante y la inminente guerra física son dos de las principales razones que habrían llevado a los hombres a constituir el Estado. Dado que no es posible encontrar empíricamente una formación social que cuente con el "estado de naturaleza" descrito en el capítulo $13^{\circ}$ del Leviathan, Hobbes utiliza la condición de la inminente guerra como ejemplo. Según Yves-Charles Zarka, el estado de naturaleza "ne correspond pas à un moment historique de l'humanité, mais consiste en une simulation théorique des comportements humains soit lorsque l'État n' existe pas encore, soit lorsqu 'il est détruit"s.

En la guerra de todos contra todos, las personas no tienen ninguna pers-

${ }^{3}$ Matteucci, Nicola, Lo Stato moderno (Bologna, Il Mulino, 1997), pp. 125-126.

${ }^{4}$ Bobbio, Norberto, Thomas Hobbes (Torino, Einaudi, 1989), pp. 45-46.

${ }^{5}$ Zarka, Yves-Charles, Hobbes et la pénsee politique moderne (Paris, Presses Universitaires de France, 1995), p. 128. 
pectiva sólida para su futuro ni cuentan con las garantías mínimas de que no serán despojadas en cualquier momento de sus bienes o derechos, incluyendo su propia vida, ya que no existe para ellas alguien que pueda darles tal garantía. La única garantía que tenemos en tal condición es nuestra capacidad de protegernos a nosotros mismos e inventar. Recurrir a algún posible criterio de justicia universal no tiene sentido, pues, como señalaba Hobbes, en ese estado, "Justice and Injustice have there no place. Where there is no common Power, there is no Law: where no Law, no Injustice"6.

Según Hobbes, toda esa coyuntura es consecuencia del hecho de que la existencia humana es "solitary, poore, nasty, brutish, and short". Es solitaria porque el hombre es incapaz de establecer vínculos duraderos e armónicos con sus semejantes; es pobre porque el que uno tenga que vivir con solo lo que es capaz de proteger dificulta cualquier posibilidad de obtener grandes posesiones de tierras u otros beneficios materiales; es sórdida porque el hombre es un ser centrado exclusivamente en la satisfacción de sus pasiones y que se guía por un "perpetual and restlesse desire of Power after power, a desire that ceaseth onely in Death"; es embrutecida porque es imposible, en esa situación, el desarrollo de un poder instrumental más calificado, lo que deja al hombre limitado a su poder original ${ }^{9}$; y es corta por ser una vida basada exclusivamente en la lucha por la supervivencia en un ambiente que es totalmente ofensivo y lleno de amenazas y en el que la muerte es algo inminente. El individualismo hobbesiano ${ }^{10}$, fundado en el concepto de estado de naturaleza, será el principal argumento para justificar la institución del Estado para enfrentar la realidad insoportable que su ausencia representa. Sin

${ }^{6}$ Hobbes, Thomas, Leviathan (London, Penguin Classics, 1985), p. 188.

${ }^{7}$ Ibíd., p. 186.

${ }^{8}$ Ibíd., p. 161.

${ }^{9}$ En el capítulo $10^{\circ}$ del Leviathan, cit. (n. 6), p. 150, Hobbes hace una distinción entre poder original (también dicho "natural") y poder instrumental: "Natural (original) power is outstandingness in the faculties of body or mind, such as extraordinary strength, good looks, prudence, practical skill, eloquence, generosity, nobility. Instrumental powers are acquired through natural powers or through luck; they are means and instruments to acquire more, for example riches, reputation, friends, and the secret working of God which men call good luck. For power is like fame in that it increases as it proceeds; or like the motion of falling heavy bodies, which go faster as they go further."

${ }^{10}$ Jaume, Lucien, Hobbes et l'Etat représentatif moderne (Paris, Presses Universitaires de France, 1986), p. 9 : “Le concept d'individualisme chez l'auteur du Léviathan devra être défini avec précision car ce terme est souvent utilisé dans un sens très extensif, et finit par signifier: qui prend l'individu pour unité de base. Or ce ne serait rendre que très imparfaitement compte de la problématique du théoricien anglais: celui-ci opère une dissociation entre l'individualism (relevant d'une anthropologie, combinée avec la théorie de l'état de nature) et l'individualité, qui s'exerce dans le cadre social et sous le règne des lois du soverain (monarque ou assemblée aristocratique ou démocratique)". 
embargo, observamos que, como argumenta Yves-Charles Zarka, la paz "ne supprime ni le différend, ni la controverse, elle permet seulement de trancher le conflit par la loi, et non par des actes de violence privée. Le passage de la guerre à la paix fait de la loi une question centrale"11.

Por otra parte, analizando la obra de Locke, vemos que él no trata de hacer consideraciones antropológicas pesimistas acerca de la conducta de los hombres cuando no están sometidos a ningún tipo de poder. Al principio, podemos ver que Locke, a diferencia de Hobbes, crea una división entre el "estado de naturaleza" y el "estado de guerra". Por "estado de naturaleza" Locke entiende aquel tiempo anterior a la formación de las sociedades civiles, cuando los hombres tenían la "perfect freedom to order their actions, and dispose of theirpossessions and persons, as they think fit, within the bounds of the law of nature, without asking leave, or depending upon the will of any other man"12.

Téngase en cuenta que aquí surge la noción de ley de la naturaleza como un elemento condicionante de la acción humana. Se trata de una ley que, por un principio de la razón, enseña a todos aquellos que la consultan que, siendo todos iguales e independientes, nadie debe dañar a otro en su vida, salud, libertad o posesiones ${ }^{13}$. Entonces, para fundamentar su idea de ley de la naturaleza, Locke se apoyará en un argumento religioso, el de la omnipotencia divina y de la subordinación de los hombres a Dios, según el cual no pueden destruirse unos a los otros porque, si así lo hicieran, eso implicaría atacar algo que es propiedad de Dios: los mismos hombres.

Cuando desarrolla su concepción de estado de guerra, Locke pone de manifiesto sus puntos de desacuerdo con Hobbes. Para el autor del Leviathan, el estado de guerra y el estado de naturaleza se confunden, ya que aquella es una guerra continua de todos contra todos. Sin embargo, Locke ve el estado de guerra como un hecho circunstancial, es decir, como algo que tiene un principio y un final. El estado de guerra se iniciaría cuando alguien declara "by word or action, not a passionate and hasty, but sedate, settled design upon another man's life puts him in a state of war with him against whom he has declared such an intention, and so has exposed his life to the other's power to be taken away by him, or any one that joins with him in his defence, and espouses his quarrel" 14 . Y solo terminaría cuando un juez o una autoridad superior fuera reconocida por las partes como capaz de resolver el caso, porque, de lo contrario, cuando la voluntad de uno tuviera que prevalecer sobre la del otro, los que fuesen partisanos del perdedor podrían buscar venganza por

\footnotetext{
${ }^{11}$ Véase Zarka, Y.-Ch., Hobbes, cit. (n. 5), p. 145.

${ }^{12}$ Locke, John, Two Treatises of Government (London, Guernsey Press, 1986), p.

${ }^{13}$ Ibíd., pp. 118-9.

${ }^{14}$ Ibíd., p. 125.
} 118. 
eso, manteniendo el estado de guerra. Se verifica así la absoluta necesidad de existencia de instituciones públicas y de un gobierno civil como medios capaces de mantener la paz y la concordia mutua.

Téngase en cuenta que Locke hace referencia a las nociones de "justo" e "injusto" en el estado de guerra, a diferencia de Hobbes ${ }^{15}$. Por lo tanto, él recurre a la ley fundamental de la naturaleza para extraer los argumentos que le permitan hablar de una acción justa en el estado de guerra, pues, de acuerdo a esa ley, el hombre debe ser preservado siempre que sea posible y se debe dar preferencia a la seguridad de los inocentes y a la de las personas sometidas a la ley común de la razón ${ }^{16}$. Según George Sabine, la doctrina di Locke "si doveva basare sull 'esatta spiegazione del significato di legge di natura, su cui si fondava la condizione pre-politica d’assistenza reciproca e secondo cui la società politica sorgeva"17.

Así, podemos ver, claramente, la distinción entre el pensamiento de Hobbes y el de Locke sobre el estado de naturaleza. Sin embargo, el propio Locke quiso subrayar esa distinción al afirmar que: "And here we have the plain difference between the state of nature and the state of war, which however some men have confounded, are as far distant, as a state of peace, good will, mutual assistance and preservation, and a state of enmity, malice, violence and mutual destruction, are one from another" ${ }^{18}$.

Aunque Locke haya expresado su intención de disociar el estado de guerra del estado de naturaleza e, implícitamente, crear una concepción fraterna, pacífica y alegre del hombre, un análisis completo de su obra revela contradicciones. En el capítulo $9^{\circ}$ de Two Treatises of Government, intitulado if the Ends of Political Society and Government, el autor escribe como si estuviera

${ }^{15}$ Hоввеs, Th., Leviathan, cit. (n. 6), p. 202: "Therefore before the names of Just, and Unjust can have place, there must be some coercive Power, to compell men equally to the performance of their Covenants, by the terrour of some punishment, greater than the benefit they expect by the breach of their Covenant; and to make good that Property, which by mutuall Contract men acquire, in recompence of the universall Right they abandon: and such power there is none before the erection of a Commonwealth".

${ }^{16}$ Locke, J., Two Treatises of Government, cit. (n. 12), p. 125: “[... it being reasonable and just, I should have a right to destroy that which threatens me with destruction: for, by the fundamental law of nature, man being to be preserved as much as possible, when all cannot be preserved, the safety of the innocent is to be preferred: and one may destroy a man who makes war upon him, or has discovered an enmity to his being, for the same reason that he may kill a wolf or a lion; because such men are not under the ties of the common law of reason, have no other rule, but that of force and violence, and so may be treated as beasts of prey, those dangerous and noxious creatures, that will be sure to destroy him whenever he falls into their power".

${ }^{17}$ SABINE, George, Storia delle dottrine politiche (traducción di Luisa de Col, $4^{\mathrm{a}}$ edición, Milano, Edizioni di Comunità, 1962), p. 423.

${ }^{18}$ Locke, J., Two Treatises of Government, cit. (n. 12), p. 126. 
inspirado por el capítulo $13^{\circ}$ del Leviathan: "If man in the state of nature be so free, as has been said; if he be absolute lord of his own person and possessions, equal to the greatest, and subject to nobody, why will hepart with his freedom? Why will he give up this empire, and subject himself to the dominion and control of any other power? To which it is obvious to answer, that though in the state of nature he hath such a right, yet the enjoyment of it is very uncertain, and constantly exposed to the invasion of others: for all being kings as much as he, every man his equal, and the greater part no strict observers of equity and justice, the enjoyment of the property he has in this state is very unsafe, very unsecure. This makes him willing to quit a condition, which, however free, is full of fears and continual dangers: and it is not without reason, that he seeks out, and is willing to join in society with others, who are already united, or have a mind to unite, for the mutual preservation of their lives, liberties and estates, which I call by the general name, property"19.

Crawford Macpherson trató de explicar esa contradicción a partir de un análisis sociohistórico ${ }^{20}$. El pensamiento de Locke fue fundamentalmente influenciado por la clase liberal burguesa, por lo que la propiedad ${ }^{21}$ fue, para él, uno de los tres derechos básicos (junto a la vida y a la libertad). Eso determinó la necesidad de encontrar una justificación racional para que la constitución del Estado no estuviera fundada en la doctrina de Hobbes, considerada demasiado apartada de las leyes morales tradicionales. En consecuencia, era necesario sustentar, basándose en la ley natural, la igualdad entre los hombres en estado de naturaleza y encontrar una explicación natural para la desigualdad, que Locke atribuye a la diferencia de racionalidad entre los hombres ${ }^{22}$. Para la satisfacción de sus contemporáneos burgueses, Locke cumplió ambas tareas.

En cambio, Rousseau tiene otra visión de la naturaleza humana. Él busca, mediante la razón, reconstruir lo que sería el hombre natural, o el hombre en estado de naturaleza. El estado de naturaleza rousseauniano representa la etapa inicial de la sociedad, caracterizada por la pureza y la integridad completa; un momento en el que las personas vivían en absoluta libertad y autonomía sin que existiera ninguna necesidad de establecer vínculos

${ }^{19}$ Ibíd., p. 179.

${ }^{20}$ Macpherson, Crawford, The Political Theory of Possessive Individualism. Hobbes to Locke (Oxford, Clarendon Press, 1964), pp. $217-9$.

${ }^{21}$ Locke, John, Two Treatises of Government, cit. (n. 12), p. 180: "The great and chief end, therefore, of men's uniting into commonwealths, and putting themselves under government, is the preservation of their property".

${ }^{22}$ Macpherson, C., The Political Theory of Possessive Individualism, cit. (n. 20), p. 221: "He also justifies, as natural, a class differential in rights and in rationality, and by doing so provides a positive moral basis for capitalist society". 
permanentes entre ellas. Aunque Rousseau trató de argumentar que estaba haciendo una reconstrucción histórica para llegar a su concepto de estado de naturaleza, toda su metodología de investigación tiene el mismo cariz teórico-hipotético adoptado por Hobbes y Locke. La única diferencia es que estos lo reconocen, pero Rousseau no.

A diferencia de Hobbes, que defiende la tesis de que el hombre es un ser naturalmente sin miedo y concentrado solamente en el combate y en la lucha, Rousseau entiende el hombre en un estado de la naturaleza -el hombre natural, para usar sus términos- como alguien que "est toujours tremblant, eprêt à fuir au moindre bruit qui le frappe, au moindre mouvement qu' il apperçoit"23. Eso sería una consecuencia del hecho de que los seres humanos poseen una tendencia natural a temer lo que no conocen en la medida en que no logran distinguir el bien y el mal físicos que ese nuevo objeto o situación les ofrece. Recién después de haber medido sus fuerzas con otros hombres, animales, o con la adversidad, será posible para el hombre atacar, pues tendrá un paradigma para hacer proyecciones en cuanto a situaciones nuevas y similares a las que eventualmente tendrá que enfrentar.

Por lo tanto, se puede ver claramente que, para ambos autores, la forma en el hombre en estado de naturaleza actuará es diametralmente distinta: Hobbes ve en la anticipación de la acción una de las más importantes formas de acción; se trata de un comportamiento activo basado en la mera suposición, que no implica necesariamente una amenaza real de la otra parte. Para Rousseau, en cambio, la conducta humana puede incluso entenderse como pasiva, pues el individuo recién actuará después de que el otro actúe y siempre que haya llegado a la conclusión de que es capaz de luchar contra el otro. El hombre natural de Hobbes es, para Rousseau, el hombre de una sociedad civil corrupta.

A pesar de las diferencias, ambos autores tienen dos puntos en común sobre la naturaleza humana: el instinto de autopreservación y la preponderancia de las pasiones. Sin embargo, aunque Rousseau desarrolle una línea de razonamiento que condiciona las acciones humanas a lo que fue determinado por las pasiones, para él, no existen pasiones innatas y las pasiones son puntos de referencia para el perfeccionamiento de la razón, lo que contradice completamente el pensamiento de Hobbes. Las pasiones serían fruto de las necesidades vitales del hombre y solo podrían desarrollarse después de manifestadas en él. En efecto, para Rousseau, no existe un conocimiento previo

${ }^{23}$ Rousseau, Jean-Jacques, Discours sur Porigine et les fondements de linégalité parmi les hommes (Paris, Gallimard, 2003), p. 66. 
a la acción que permita hablar de la existencia y manifestación de cualquier tipo de pasión que no haya sido provocada por el componente material ${ }^{24}$.

En una nota en Discours sur l'origine et les fondements de l'inégalitéparmi les hommes, Rousseau complementa el razonamiento anterior: "Excepté le seulnecessaire Physique, que la Nature même demande, tous nos autres besoins ne sont tels que par l'babitude avant laquelle ils n'étoient point des besoins, ou par nos desirs, et l'on ne desire point ce qu'on n'est pas en état de connaitrê. $D$ 'où il suit que l' homme sauvage ne desiderant que les choses qu'il connoît et ne connossaint que celles dont la possession est en son pouvoir ou facile à acquerir, rien ne doit être si tranquille que son ame et rien si borné que son esprit" ${ }^{25}$.

Este último razonamiento demuestra la realidad del hombre natural, es decir, la realidad de vivir en un ambiente tranquilo y sin conflictos, dado que sus necesidades son limitadas y no tiene motivos para un conflicto importante. Esa realidad es completamente opuesta a lo que concibe Hobbes, un ambiente hostil en el que el conflicto y la búsqueda de más y más poder son características esenciales.

Así, el hombre natural de Hobbes es, para Rousseau, el hombre de una sociedad civil corrupta, pues Rousseau cree que es en el momento de formación de la sociedad que el hombre comienza un proceso de deterioración de su condición de pureza innata; el desarrollo de ese proceso podría conducir a su descaracterización (o desnaturalización) y al nacimiento de un individuo con características fundamentalmente diferentes a las que tenía cuando se encontraba en su estado natural ${ }^{26}$.

José F. Fernández Santillán pone de relieve el hecho que, para Rousseau, la

${ }^{24}$ Ibíd., p. 73 : “Quoiqu'en disent les Moralistes, Pentendement humain doit beaucoup aux Passions, qui, d'un commun aveu, lui doivent beaucoup aussi: c'est par leur activité, que notre raison se perfectionne; Nous ne cherchons à connoître, que parce que nous desirons de joü̈r, et iln'est pas possible de concevoir pourquoi celui qui n' auroit ni desirs ni craintes se donneroit la peine de raisonner. Les Passions, à leur tour, tirent leur origine de nos besoins, et leur progrès de nos connoissances; car on ne peut désirer ou craindre les choses, que sur les idées qu'on en peut avoir, ou par la simple impulsion de la Nature; et l'homme Sauvage, prive de toute sorte lumiéres, n'éprouve que les Passions de cette derniére espéce; Ses desirs ne passent pas ses besoins Physiques".

${ }^{25}$ Ibíd., p. 141.

${ }^{26}$ Ibíd., p. 52 : "Semblable Glaucus, que le tems, la mer et les orages avoient tellement défigurée, qu'elle ressembloit moins à un Dieu qu'à une Bête féroce, l' ame humaine altérée au sein de la société par mille causes sans cesse renaissantes, par l acquisition d'une multitude de connoissances et d'erreurs, par les changemens arrivés à la constitution des Corps, et par le choc continuel des passions, a, pour ainsi dire, chargé d'apparence au point d'être presque méconnoissable et lon n'y retrouve plus, au lieu d'un être agissant toûjours par des Principes certains et invariables, au lieu de cette Celeste et majestueuse simplicité dont son Auteur lavoit empreinte, que le difforme contraste de la passion qui croit raisonner et de lentendement a delire". 
sociedad civil se convierte en un problema, en vez de constituir una solución; para resolver el problema de la sociedad civil será necesario el establecimiento de la Commonwealth ${ }^{27}$.

\section{LA FORMACIÓN DEL CONTRATO SOCIAL}

Después de estas consideraciones sobre la naturaleza humana, se pasa al análisis de la formación del contrato social en los tres autores estudiados.

En la filosofía política de Hobbes el proceso de establecimiento de un Soberano tiene dos fases. La primera se caracteriza por la horizontalidad de la relación, cuando el acuerdo se hace entre cada individuo y su similar. El segundo momento es caracterizado por la verticalidad: se basa en el permiso otorgado por todos a una persona, o asamblea, para el ejercicio ilimitado del poder en su nombre. En pocas palabras, el contrato social es un acuerdo general entre todos los sujetos para que una persona, o asamblea, actúe como si fuera cada individuo ejerciendo su propio poder. Por lo tanto, el establecimiento del Soberano tiene estrecha relación con el contrato social. Más que un mero acuerdo, el contrato social es de carácter obligatorio entre las partes, pues mantiene el poder coercitivo del Soberano, lo que no sería necesario -o al menos deseable- en un mero acuerdo respaldado únicamente por la moral. Cuando dos o más personas están de acuerdo sobre un tema determinado, el elemento subjetivo que apoya la existencia y el cumplimiento de ese acuerdo es sustancialmente la moral. Sin embargo, la constitución del contrato social no tiene ese elemento subjetivo como base única de su eficacia, sino la fuerza procedente de un poder soberano creado para hacer cumplir el contrato y hacer que se ejecuten sus términos en la realidad material ${ }^{28}$.

${ }^{27}$ Santillán, José, Hobbes y Rousseau. Entre la autocracia y la democracia. (Mexico, D. F., Fondo de Cultura Económica, 1988), p. 60.

${ }^{28}$ Gauthier, David, Hobbes's Social Contract, en Morris, Christopher (editor), The Social Contract Theorists: Critical Essays on Hobbes, Locke and Rousseau (Lanham, Rowman \& LittleField Publishers, 1999), p. 61, confirma esa idea: "I propose to distinguish contracts from other agreements by characterizing the former as exchanges of intentions to act that introduce incentives, wheter internal or external, moral or other, to supplement or replace each party's motivation to attain the true objective of the agreement. In particular, I distinguish contracts from purely coordinative agreement. Both require that each person prefers the outcome of agreement to that of no agreement. In purely coordinative agreements, each also prefers compliance by her fellows, and this preference for compliance is not induced by sanctions, external or internal. Furthermore, each expects the others to comply, and intends to comply herself. No part of the agreement exists solely to ensure compliance with the remainder, so that, leaving compliance aside, omitting any of the terms of the agreement would be regarded as undesirable by at least on of the parties". 
Si la soberanía solo se centrase en la motivación de las personas para colaborar para el cumplimiento de la ley y la manutención de la seguridad social, ese poder instituido estaría irremediablemente destinado al fracaso. La expectativa de obtener una respuesta activa de los súbditos debe darse en la medida que ellos estén dispuestos a colaborar; así, no cabe al súbdito, por ejemplo, interferir en el castigo impuesto por el soberano a quien haya violado la ley y compete a él estar dispuesto a concordar con las órdenes del soberano. Todas esas atribuciones del súbdito deben tener como objetivo el mantenimiento de su propia seguridad; no obstante eso, en el momento en que sea necesario auxiliar al soberano, cuando este lo convoque, ya se le pedirá algo que puede poner en peligro la seguridad del propio súbdito. Si se debe arrestar a un forajido, el sentido de la autoconservación individual del hombre o de los hombres que lo perseguirán debe dar lugar a la voluntad de mantener el bien mayor de la colectividad, es decir, la seguridad de todos, aunque entre ellos haya muchos que no pondrían en riesgo su seguridad personal para realizar dicha tarea.

Según la psicología social hobbesiana, para que su "existir" no sea algo efímero, el hombre se siente en la obligación de contratar, no por respeto a su comunidad o por querer ver el progreso y desarrollo de esta, sino porque su propia existencia se encuentra amenazada y la posibilidad de grandes logros personales está obstaculizada por la condición natural de la humanidad en el estado de naturaleza ${ }^{29}$.

El proceso de abnegación de sus propios derechos y libertad en beneficio del Soberano es el producto máximo del egoísmo de alguien que entiende que la vida es insostenible en un estado de naturaleza y que desea establecer, junto a sus semejantes, un aparato institucional superior a ellos y que sea capaz de asegurar que los logros personales y la vida de cada uno no serán ofendidos impunemente. En suma, Hobbes cree que el acto de institución estatal (así como todas las demás acciones que el hombre hace) es un acto fundamentalmente egoísta, racional y que contempla el bien común solo a título de objetivo secundario ${ }^{30}$.

Si volvemos nuestra mirada hacia Locke, nos damos cuenta de que él siguió un camino diferente al trazado por la fórmula propuesta por Hobbes para estudiar los aspectos antropológicos y psicológicos del hombre. Sin em-

${ }^{29}$ Johnston, David, The Retoric of Leviathan (Princeton, Princeton University Press, 1989), p. 101: "Fear of death is more than a merely reasonable or admissible ground of argument within Hobbes's political theory. It is the basis of his justification of the scope of sovereign authority and of his view of the irreducible liberty of subjects".

${ }^{30}$ JaUme, L., Hobbes et l'Etat représentatif moderne, cit. (n. 10), p. 26 : "Il faut chercher dans la structure de l'Etat les possibilités de limitation de l'individualisme illimité, pour que l'unité s'instaure en politique, et la sécurité dans la vie privée". 
bargo, y como ya lo hemos visto, Locke presentó aporías en su justificación racional del estado de naturaleza que no lo distanciaron de Hobbes con toda la solidez que él quería.

Locke argumentaba que en el estado de naturaleza existían derechos de propiedad, pero era absolutamente inviable mantener tales derechos protegidos en un ambiente desprovisto de una sociedad política organizada (Commonwealth) -que tenía en la conservación de la propiedad su principal fin- y, sobre todo, sin la presencia de un poder imparcial, soberano y capaz de garantizar la protección de tales derechos; la instrumentalización de ese poder soberano se daría por medio de la creación de los poderes legislativo, judicial y ejecutivo ${ }^{31}$.

Existen algunas peculiaridades ${ }^{32}$ en el estado de naturaleza en sí mismo y que Locke postula como causas de la formación de una sociedad política o, mejor dicho, de un Estado: $i$ ) ausencia de una ley establecida, reconocida por todos y eficaz en el logro de su objetivo; $i i$ ) ausencia de un juez imparcial para resolver los conflictos y encontrar soluciones para los conflictos entre los individuos, garantizando que ninguna de las partes pague por los daños que no haya causado; iii) ausencia de un poder suficientemente fuerte para hacer cumplir las sentencias. Locke entiende que los hombres, cuando vencidos en una disputa, buscan por todos los medios resistir a los efectos de la sentencia que los perjudiquen.

El discurso de Locke se distinguirá de la filosofía hobbesiana acerca de las causas de institución de un Estado (o Commonwealth, como lo prefiere Locke). Observamos que, cuando se analiza al hombre en estado de naturaleza, para Hobbes, todos los derechos están en constante amenaza, mientras que, para Locke, solo la propiedad se encuentra bajo la amenaza perenne y continua de violación por parte de sus semejantes, aunque, como ya lo hemos visto, Locke se contradijo al abordar esa cuestión. Según él, el Estado intentaría, en primer lugar, asegurar el derecho natural a la propiedad que cada

${ }^{31}$ Fioravanti, Maurizio, Costituzione (Bologna, Il Mulino, 1999), pp. 90-1: “Agli uomini mancava però quella che lo stesso Locke chiamava una standing rule, una regola fissa e consolidata, capace di preservare nel tempo la property già acquisita nello stato di natura. Per questo, gli uomini decidevano di uscire dallo stato di natura e d'istituire la società politica. In essa quegli uomini vedevano essenzialmente uno strumento di perfezionamento della condizione già esistente, che consentiva di mettere a servizio della medessima property, dei loro diritti alcune istituzioni politiche che come tali mai avrebbero potuto stabilirsi nello stato di natura: un legislatore e una legge capace di rappresentare la 'misura comune' nella determinazione del torto e della ragione nelle controversie tra gli individui, un giudice 'certo e imparziale', su cui poter sempre contare per l'applicazione della legge, e un potere ulteriore, quello esecutivo, che abbia in sé, in modo incontestabile, la forza necessaria a far eseguire le sentenze".

${ }^{32}$ Locke, J., Two Treatises of Government, cit. (n. 12), pp. 179-82. 
hombre tiene, mientras que, para el autor del Leviathan, el Estado surge con la tarea principal de establecer la paz social, impidiendo así que el derecho natural a la vida sea violado banalmente por otro hombre.

\section{LAS FORMAS DE ESTAdo Y DE GOBIERNo}

Una distinción fundamental entre los dos autores se puede constatar en la su concepción sobre el primer paso siguiente a la formación del Estado, es decir, sobre el momento de elección de la formas de Estado y de Gobierno.

La predilección de Hobbes por la monarquía absoluta es un rasgo característico de su filosofía política; el autor relega el asambleísmo al segundo lugar en preferencia. La figura del monarca era la que más se ajustaba a la autoridad política ilimitada que debería concentrar en sí misma el poder para justificar la legitimidad de cualquier regla jurídica, moral o religiosa, teniendo siempre como fin la búsqueda de la paz social. Antes de ser bien gobernado, el Estado debía ser gobernable. La ley surgiría como positivación de la recta razón (right reason) de quien (o quienes) tiene el poder para concebirla, representando así la primacía de la autoridad en detrimento de la sabiduría: "non veritas sed auctoritas facit legem" 33 . La racionalidad hobbesiana tenía en el monarca -y la posibilidad de que él fuera la persona con las condiciones más adecuadas para desarrollar su recta razón- el argumento central para invalidar la fundamentación meramente hereditaria de la monarquía, entonces vigente, e intentar encontrar en el absolutismo del poder soberano la mejor manera de poner fin al estado de guerra que caracteriza la condición natural del hombre. Para Hobbes, cualquier intento de dividir el poder soberano o de transferir sus prerrogativas a otros órganos tendría como resultado la destrucción del Estado. Todo el poder de los organismos no estatales debería emanar, por subordinación o por creación, del poder soberano ${ }^{34}$.

En otro sentido, y, como Hobbes, muy influido por las condiciones sociohistóricas de su época, Locke demuestra oponerse a la monarquía absoluta, sobre todo por entender que el poder despótico es un poder absoluto y arbitrario que va más allá de los límites de lo acordado en la formulación del contrato social; tanto sería así que ni siquiera la misma naturaleza con-

\footnotetext{
${ }^{33}$ Hoвbes, Thomas, Dialogue Between a Philosopher and a Student of the Common Law of England (Chicago, Cropsey, 1998).

${ }^{34}$ JELLINEK, Georg, L'état modern et son droit, I: Théorie générale de létat (traducción de George Fardis, Paris, Panthéon Assas, 2005), p. 112 : "Pour lui, toute distinction d'un seul des droits constituent la souveraineté et toute concession de ces droits à un autre qu'au titulaire du summum imperium, aurait pour effet de détruire l'État. Tout pouvoir des corps politiques non étatiques, est la creation du pouvoir souverain et lui est subordonné".
} 
sintió que el hombre permitiera que otros hombres ofendieran su vida en el momento que más les conviniera. El único momento en el que el hombre pierde su derecho a la vida es cuando se declara en estado de guerra contra un semejante ${ }^{35}$. Según Locke, el defecto fundamental que manchaba la monarquía surgió de su absoluta incompatibilidad -como de cualquier otro modelo político dotado de un solo centro de poder- con la organización de una sociedad civil bien ordenada ("well ordered Commonwealth"), ya que la confusión de poderes en un mismo cuerpo haría el Ejecutivo y el Legislativo inseparables y sin ninguna relación de continuidad entre sus actos ${ }^{36}$.

Sin embargo, cabe recordar que Locke, junto con Montesquieu, fue uno de los principales idealizadores de la separación de poderes; por eso, para él, el principal momento después de la formación del Estado es el de estructuración del Poder Legislativo, ya que ese será el órgano encargado de la formulación de las leyes que regirán las relaciones sociales y, sobre todo, garantizarán la propiedad, estando los otros poderes a él subordinados. El Poder Ejecutivo tendría un carácter eminentemente administrativo y de ejecución de las leyes, pues la elaboración de dichas leyes ocurriría exclusivamente en el Parlamento. De esa forma, queda en franca evidencia el carácter supremo del órgano legislativo ${ }^{37}$.

Para la historia del liberalismo político y del constitucionalismo moderno, la contribución más significativa y original presentada por el pensamiento de Locke fue, según Maurizio Fioravanti, "la fondamentale distinzione tra potere assoluto e potere moderato" ${ }^{38}$. Es a partir de esa noción de poder moderado que el concepto de soberanía en Locke puede encontrar límites internos a su ejercicio, diversamente de lo verificado tanto en Hobbes como en Rousseau, que entendieron la soberanía como una prerrogativa que, en esencia, desconoce límites. Mientras que para esos dos últimos filósofos los derechos de los individuos no podrían prevalecer cuando estuvieran en conflicto con el poder del Soberano (Sovereign) o con la voluntad general del pueblo ("volonté generale du peuple"), para Locke, los derechos de los individuos forman en sí mismos una limitación natural al poder del gobierno,

${ }^{35}$ Locke, J., Two Treatises of Government, cit. (n. 12), pp. 185-6.

${ }^{36}$ Fioravanti, M., Costituzione, cit. (n. 31), pp. 91-2.

${ }^{37}$ Locke, J., Two Treatises of Government, cit. (n. 12), pp. 183-4: "The great end of men's entering into society, being the enjoyment of their properties in peace and safety, and the great instrument and means of that being the laws established in that society; the first and fundamental positive law of all commonwealths is the establishing of the legislative power; as the first and fundamental natural law, which is to govern even the legislative itself, is the preservation of the society, and (as far as will consist with the public good) of every person in it. This legislative is not only the supreme power of the common-wealth, but sacred and unalterable in the hands where the community have once placed it".

${ }^{38}$ Fioravanti, M., Costituzione, cit. (n. 31), p. 92. 
una vez que la búsqueda de protección de tales derechos fue lo que justificó la creación del Estado $^{39}$. Además, en el modelo de Locke, la soberanía ya no tiene como referencia a una sola persona, ni siquiera al pueblo soberano, sino "un sistema di poteri tenuti in equilibrio dalla costituzione" ${ }^{\text {"40 }}$. La soberanía pierde, así, su carácter absoluto y se convierte en la representación de un conjunto de poderes soberanos autorizados por la Constitución ${ }^{41}$.

Con respecto a Rousseau, a medida que avanzamos en su pensamiento político, se observa que, basándose en sus consideraciones sobre el hombre en estado de naturaleza, su justificación del contrato social será sustancialmente diferente de la producida por Hobbes y Locke. Para Rousseau, aunque el hombre no sea violento y egoísta por naturaleza, el desarrollo de sus relaciones interpersonales conducirá inexorablemente a un aumento de las restricciones a la libertad de cada individuo. La pureza inicial que tenía cuando en estado de naturaleza disminuye progresivamente a medida que él y sus compañeros perfeccionan de manera desigual sus posibilidades individuales, lo que hace que se superpongan unos a los otros y conquisten los bienes de manera desigual. Esa desigualdad natural sería una de las principales causas responsables de generar restricciones a la libertad de los hombres.

El problema fundamental de la existencia humana gira en torno a la libertad; y el contrato social es el instrumento que los hombres tienen para constituir un Estado capaz de mantenerlos tan libres como antes ${ }^{42}$.

Los modelos de contrato social adoptados por Hobbes y Rousseau se distinguen en cuanto a la posición del individuo en relación con el Estado (o la sociedad civil): para Hobbes, el individuo no debe hacer otra cosa que obedecer las órdenes del soberano, mientras que, para Rousseau, los ciudadanos deben participar en el proceso de toma de decisiones. La idea de bien

${ }^{39}$ Véase Simonutti, Luisa, La souveraineté come problème chez Locke, en CAZZaniga, Gian Mario - Zarka, Yves-Charles (editores), Penser la souveraineté (Paris, Vrin, 2001), pp. 154-5.

${ }^{40}$ Fioravanti, Maurizio, Costituzione e popolo sovrano. La Costituzione italiana nella storia del costituzionalismo moderno (Bologna, Il Mulino, 2004), p. 61.

${ }^{41}$ Ibíd., p. 61: "A rigore, si potrebbe dire che non c’è più 'sovranità', ma solo esercizio di poteri sovrani che sono tali in quanto autorizzati dalla costituzione. Più prudentemente si potrebbe anche dire che il modello ora esaminato ci mostra il rovescio della nostra medaglia: non più la costituzione come prodotto di un atto sovrano, ma anche l'opposto, la sovranità come prodotto e risultato dell'operare concreto della norma costituzionale".

${ }^{42}$ Rousseau, Jean-Jacques, Contrat Social (Paris, Gallimard, 2002), p. 182 : “Trouver une forme d'association qui defende et protege de toute la force commune la personne et les biens de chaque associé, et par laquelle chacun s'unissant à tous n'obéisse pourtant qu'à lui-même et reste aussi libre qu'apavarant? Tel est le problême fondamental dont le contract social donne la solution". 
común requiere que cada individuo se transforme en una parte activa de la totalidad $^{43}$. La centralización que, para Hobbes, supone el poder absoluto del monarca se convierte, en el pensamiento de Rousseau, en una centralización equitativa del poder, pero no en las manos de una sola persona: según el ginebrino, el pueblo sería el legítimo titular del poder soberano sobre su propia comunidad política ${ }^{44}$. En similar sentido, Nicola Matteucci sustenta que, para Rousseau, "è il popolo stesso a autogovernarsi dandosi direttamente le leggi, senza alcuna mediazione di rappresentanti, mentre il governo in senso stretto ha il mero compito di applicare le leggi e quinda dá forza a una volontà altrui" 45 .

Mientras que, para Hobbes, la razón que justifica la institución del Estado es la protección de la vida, para Locke, es la protección de la propiedad y, para Rousseau, el argumento principal será el de la garantía de la libertad. Se trata de una libertad convencional que viene para sustituir la libertad natural, pero cuyos efectos deben ser de la misma intensidad, so pena de estarse constituyendo un Estado contrario a la naturaleza humana.

Para evitar que algunos hombres tengan ventajas sobre los demás, Rousseau cree que la voluntad general ${ }^{46}$ debe formar un cuerpo colectivo soberano dentro del cual la libertad sea mantenida por medio de la noción de igualdad que existe en el grupo, lo que impediría que un individuo pudiera violar la libertad de los demás sin sufrir algún tipo de represión político-institucional. Rousseau sostenía que "chacun se donnant à touts ne se donne à personne, et comme il n'y a pas une associé sur lequel on n'acquiere le même droit qu'on lui cede sur soi, on gagne léquivalent de tout ce qu'on perd, et plus de force pour conserver ce qu'on a" ${ }^{47}$. Eso determina que la soberanía popular en Rousseau sea "incondizionata non meno di quanto lo fosse, in autori come Bodin e Hobbes, la sovranità del monarca assoluto" ${ }^{4}$.

Aunque en el momento de formulación del contrato social todos los derechos naturales de la comunidad se estén enajenando sin reservas, el hombre así procede para poder disfrutar, en seguida, de los mismos derechos por medio de la fuerza del cuerpo político. En otras palabras, la fuerza que hace

${ }^{43}$ Santillán, J., Hobbes y Rousseau, cit. (n. 27), p. 88.

${ }^{44} I$ bíd., p. 135: "La tarea reservada por Hobbes para uno solo (el príncipe), en Rousseau es desempeñada por todos (el pueblo); en Hobbes el príncipe manda y todos los demás obedecen, en Rousseau todos mandam y todos obedecen”.

${ }^{45}$ Matteucci, N., Lo Stato moderno, cit. (n. 3), p. 111.

${ }^{46}$ Rousseau, J.-J., Contrat Social, cit. (n. 42), p. 191 : "Pour qu'une volonté soit générale il n'est pas toujours nécessaire qu'elle soit unanime, mais il est nécessaire que toutes les voix soient comptées; toute exclusion formelle rompt la géneralité".

${ }^{47}$ Ibíd., p. 183.

${ }^{48}$ Zolo, Danilo, Isignori della pace (Roma, Carocci, 1998), p. 114. 
efectivos los derechos que el hombre ejercía por sí mismo en la naturaleza deja de ser meramente individual y pasa a ser una fuerza que surge del poder del cuerpo político y que encuentra en la ley un instrumento definitivo para la preservación de ese cuerpo ${ }^{49}$.

Con esas posiciones Rousseau demuestra ser partidario de un modelo de Estado republicano y democrático. Su aversión a la monarquía se pone de manifiesto cuando afirma que: "un defaut essenciel e inévitable, qui mettra toujours le gouvernement monarchique au dessous du républicain, est que dans celui-ci la voix publique n'éleve presque jamais aux premieres places que des hommes éclaires et capables, qui les remplissent avec honneur: au lieu que ceux qui parviennent dans les monarchies ne sont le plus souvent que de petits broullions, de petits fripons, de petits intrigans, à qui les petits talens qui font dans les Cours parvenir aux grandes places, ne servent qu'à montrer au public leur ineptie aussi-tôt qu'ilsy sont parvenus" $"$.

\section{LA GÉNESIS DE LAS CARACTERÍSTICAS DE LA SOBERANÍA NACIONAL}

Una vez analizadas las concepciones de hombre natural y también los fundamentos que legitiman la creación de un Estado civilmente organizado en las filosofías de Hobbes, Locke y Rousseau, alcanzamos el punto final de este breve estudio comparativo: demostrar la génesis de la indivisibilidad e de la inalienabilidad de la soberanía en la tradición contractualista formada por esos tres autores. No obstante las variaciones teóricas significativas existentes entre ellos, vemos que la defensa del principio de soberanía fue preservada de modo unívoco entre los más importantes contractualistas de la filosofía política.

Hobbes afirmaba que la soberanía es una autoridad dada por cada individuo (contratante) al Estado (contratista), como resultado de una autorización que el primero le da al último para actuar como si fuera el propio individuo, atribuyéndole "the use of so much Power and Strength conferred on him, that by terror thereof, he is inabled to forme the wills of them all, to Peace at home, and mutuall ayd against their enemies abroad"s1. Por lo tanto, el pacto no se puede deshacer ni sustituir por otro, ni tampoco el Estado puede dividir o disponer de facultades que le atribuyeron aquellos que fueron los responsables por su formación.

Al hablar en una sociedad política como "cuerpo único" y con "poderes

${ }^{49}$ TARELlo, Giovanni, Storia della cultura giuridica moderna (Bologna, Il Mulino, 1976), pp. 328-329.

${ }^{50}$ Rousseau, J.-J., Contrat Social, cit. (n. 42), p. 232.

${ }^{51}$ Hоввеs, Th., Leviathan, cit. (n. 6), pp. 227-228. 
políticos supremos", Locke también confirma la tesis de que el poder soberano es indivisible e inalienable. A pesar de haber hecho varias referencias en su trabajo a la posibilidad de desobediencia civil y desconstitución del pacto social, dividir el poder soberano o transferir prerrogativas que son inherentes a la soberanía no pueden considerarse formas de desobediencia civil o desconstitución del contrato social, una vez que el Estado sigue existiendo, independientemente de una posible pérdida de poderes. El acto de transferencia estatal de poderes que provienen de la soberanía o de la división interna de poderes no impide que el contrato social siga teniendo efectos e incluso realizando sus objetivos iniciales. La desobediencia civil, según Locke, no debería tener propósitos sediciosos, ya que es una facultad que el individuo tiene para resistir y reaccionar contra el monarca que haya transgredido sus limitaciones constitucionales, pudiendo también ser utilizada "contro la suprema autorità del legislatore, quando questo abbia violato le vite, le libertà, ed i beni degli individui che ad esso erano stati affidati" ${ }^{2}$.

Rousseau, por su parte, dedicó el primer capítulo del libro II del Du contrat social para argumentar que "La souveraineté est inalienable", y el capítulo siguiente de la misma obra para sostener que "La souveraineté est indivisible". Según él : "la souveraineté n'étant que l'exercise de la volonté generale ne peut jamais s'aliéner, et que le souverain, qui n'est qu'un être collectif, ne peut être représenté que par lui-même; le pouvoir peut bien se transmettre, mais non pas la volonte" 53 . La soberanía es indivisible también porque "la volonté est générale, ou elle ne l'est pas; elle est celle du corps du peple, ou seulement d'une partie"54. La voluntad general que surge del cuerpo político no admitirá concurrente.

\section{Consideraciones FinAles}

A pesar de las variaciones existentes en las concepciones de soberanía desarrolladas por los tres autores, se puede concluir que la necesidad de establecimiento de un Estado soberano con poderes indivisibles, inalienables e ilimitados fue la fuerza impulsora del contractualismo político moderno. Sin cuestionar la existencia del poder, pero sí su dinámica interna y sobre todo su intensidad, vemos que el debate entre el poder moderado y el poder absoluto, a saber, la confrontación teórica que opone a Locke y Rousseau con respecto a Hobbes, prosiguió después del final de la Revolución Francesa, en particular durante el siglo XX, tras la consagración de la doctrina de los derechos humanos en las relaciones internacionales.

\footnotetext{
${ }^{52}$ Fioravanti, Maurizio, Stato e Costituzione. Materiali per una storia delle dottrine costituzionali (Torino, Giappichelli, 1993), p. 160.

${ }^{53}$ Rousseau, J.-J., Contrat Social, cit. (n. 42), p. 190.

${ }^{54}$ Ibíd., p. 191.
} 


\section{BiBLIOGRAFÍA}

Boвbio, Norberto, Thomas Hobbes (Torino, Einaudi, 1989).

Bodin, Jean, Les six livres de la République (Geneve, 1629).

Fioravanti, Maurizio, Costituzione (Bologna, Il Mulino, 1999).

Fioravanti, Maurizio, Costituzione e popolo sovrano. La Costituzione italiana nella storia del costituzionalismo moderno (Bologna, Il Mulino, 2004).

Fioravanti, Maurizio, Stato e Costituzione. Materiali per una storia delle dottrine costituzionali (Torino, Giappichelli, 1993).

Gauthier, David, Hobbes's Social Contract, in Morris, Christopher W. (coord.), The social contract theorists: critical essays on Hobbes, Locke and Rousseau (Lanham, Rowman \& LittleField Publishers, 1999).

Gauthier, David, Logic of Leviathan (Oxford, Clarendon Press, 1969).

Hobies, Thomas, De cive (Oxford, Clarendon, 1983).

Hoвbes, Thomas, Dialogue Between a Philosopher and a Student of the Common Law of England (Chicago, Cropsey, 1998).

Hobbes, Thomas, Leviathan (London, Penguin Classics, 1985).

Jaume, Lucien, Hobbes et l'Etat représentatif moderne (Paris, Presses Universitaires de France, 1986).

Jellinek, Georg, L'état modern et son droit, I : Théorie générale de létat (traducción de George Fardis, Paris, Panthéon Assas, 2005).

Johnston, David, The Retoric of Leviathan (Princeton, Princeton University Press, 1989).

Locke, John, Two Treatises of Government (London, Guernsey Press, 1986).

Macpherson, Crawford, The Political Theory of Possessive Individualism. Hobbes to Locke (Oxford, Clarendon Press, 1964).

Matteucci, Nicola, Lo Stato moderno (Bologna, Il Mulino, 1997).

Ménessier, Thierry. Principautéet souverainetéchez Machiavel, en Cazzaniga, Gian Mario - Zarka, Yves-Charles (editores), Penser la souveraineté (Paris, Vrin, 2001).

Rousseau, Jean-Jacques, Contrat Social (Paris, Gallimard, 2002).

Rousseau, Jean-Jacques, Discours sur lorigine et les fondements de l'inégalitéparmi les hommes (Paris, Gallimard, 2003).

Sabine, George H., Storia delle dottrine politiche (traducción di Luisa de Col, $4^{a}$ edición, Milano, Edizioni di Comunità, 1962).

Santillán, José F. F., Hobbes y Rousseau. Entre la autocracia y la democracia (México, D.F., Fondo de Cultura Económica, 1988).

Simonutti, Luisa, La souveraineté come problème chez Locke, en Cazzaniga, Gian Mario - Zarka, Yves-Charles (editores), Penser la souveraineté(Paris, Vrin, 2001).

TARELlo, Giovanni, Storia della cultura giuridica moderna (Bologna, Il Mulino, 1976).

Zarka, Yves-Charles, Hobbes et la pénsee politique moderne (Paris, Presses Universitaires de France, 1995).

Zolo, Danilo, I signori della pace (Roma, Carocci, 1998). 



\section{Advertencia}

La Dirección de la Revista de Derecho de la Pontificia Universidad Católica de Valparaiso deja constancia que el artículo aparecido entre las pp. 391-397 del volumen 19 (1998) de la por entonces Revista de Derecho de la Universidad Católica de Valparaiso, firmado por Christian Grollmus Fritz, bajo el título "Edificación en suelo ajeno sin previo contrato" ofrece ostensibles coincidencias textuales con el firmado por don René Ramos Pazos bajo el título "Edificación en suelo ajeno sin previo contrato", incluido en la Revista de Derecho de la Universidad de Concepción, año 53, 178 (julio-diciembre de 1985), pp. 141-147, hecho que solo muy recientemente ha sido puesto en conocimiento de la dirección de la primera de las revistas nombradas, por lo cual se ha mandado insertar esta "Advertencia" y tachar el artículo de 1998 de toda versión electrónica del mencionado volumen 19.

Valparaíso, 5 de enero de 2015. 


